\title{
A cadmium-zinc-telluride crystal array spectrometer
}

\author{
William Quam, Thomas DeVore, Harold McHugh, Robert Vogle, John Wesolowski \\ Bechtel Nevada, Special Technologies Laboratory, Santa Barbara, CA 93111
}

\begin{abstract}
This paper describes a gamma detector employing an array of eight cadmium-zinc-telluride (CZT) crystals configured as a high resolution gamma ray spectrometer. This detector is part of a more complex instrument that identifies the isotope, displays this information, and records the gamma spectrum. Various alarms and other operator features are incorporated in this battery operated rugged instrument.
\end{abstract}

The CZT detector is the key component of this instrument and will be described in detail in this paper. We have made extensive spectral measurements of the usual laboratory gamma sources, common medical isotopes, and various Special Nuclear Materials (SNM) with this detector. Some of these data will be presented as spectra. We will also present energy resolution and detection efficiency for the basic 8-crystal array. Additional data will also be presented for a 32-crystal array.

The basic 8-crystal array development was completed two years ago, and the system electronic design has been improved recently. This has resulted in significantly improved noise performance. We expect to have a much smaller detector package, using 8 crystals, in a few months. This package will use flip-chip packaging to reduce the electronics physical size by a factor of 5 .

Keywords: CZT, gamma spectrometer, isotope identifier

\section{MECHANICAL DESIGN}

The detector package, shown in Figures 1 through 3, contains the 8-CZT crystals, the analog electronics, and the high voltage power supply. The crystals are arranged in pairs in parallel into one of four FETs. This simplifies the electronics somewhat and has been shown to degrade the energy resolution by an acceptably small amount.

The CZT crystals are sandwiched between two multilayer PC boards. One of these PC boards contains a CockcroftWalton high voltage supply on one side, and pads for contact with one electrode of the CZT crystals on the other side. The second PC board has the analog electronics and output connectors on one side, and pads for contact with the other CZT electrode. An egg-crate-shaped plastic holder is used to center the CZT crystals on the contact pads. Both PC boards are rigidly spaced apart by the aluminum frame surrounding them. One side of this aluminum frame has a thin aluminum cover when incorporated into a complete instrument and the other side connects directly to the multi-channel analyzer (MCA) and CPU boards making up this instrument.

This detector module has been tested for mechanical shock and has survived repeated drops from 10 feet to a concrete floor. The aluminum housing suffered cosmetic damage but the detector operated without degradation after this testing. The complete module weighs 85 grams and this light weight minimized shock damage. The complete instrument, in its plastic housing with batteries, has survived numerous drops from 3 feet to thinly carpeted concrete floors.

\section{ELECTRONIC DESIGN}

The electronics have been optimized for low power in keeping with the battery operation of the complete instrument. A single FET is used as the charge input device for each pair of CZT crystals. Each crystal of this pair is selected for thickness, energy resolution, and relative gain using a simple test jig. We have found significant variations in 'gain' from one crystal to another and the selection process produces crystal pairs adequately matched with about $10 \%$ rejection from a large lot of crystals. Simple shaping with a 1 microsecond time constant is used in the linear amplifiers. We do not use pulse shape analysis. The loss in efficiency is significant, and a reduction in power due to fewer parts is welcome. The detector module, including the high voltage power supply, uses $+6 \mathrm{~V}$ at $160 \mathrm{~mA}$. 
Each crystal pair produces a spectrum that is stored independently of the other three spectra. An initial calibration of a detector module, using ${ }^{241} \mathrm{Am},{ }^{57} \mathrm{Co}$, and ${ }^{133} \mathrm{Ba}$ sources, produces a gain (in $\mathrm{keV} / \mathrm{ch}$ ) and an offset (in $\mathrm{keV}$ ) from a least squares fit for each of the four spectrometer channels. These data are stored in RAM and are used by the control program to digitally sum the four spectra, corrected for the gain and offset variations for each of the four channels A temperature sensor within the module is used to provide an overall gain correction with normalization to a calibration temperature. The resulting spectrum is then used for isotope identification.

\section{RADIOLOGICAL PERFORMANCE}

\subsection{8-crystal array}

Figures 4 through 10 present spectra obtained with the 8-crystal detector module in the battery powered instrument. The crystals used for these measurements were obtained from eV Products and are 5 x 5 x $5 \mathrm{~mm}$ cap electrode crystals. They were selected as noted above from various lots of crystals with the generic specification of $<4 \% \mathrm{FWHM}$ at ${ }^{57} \mathrm{Co}$. These measurements and other medical isotope data were used to construct the usual FWHM resolution vs. energy curve. This curve is shown in Figure 11. The same data were used to construct the efficiency curve shown in Figure 12.

The resolution data at FW.02M were also obtained from the same set of spectra. This width encloses most of the counts associated with a gamma peak. Some are lost due to the low energy tailing. If we use this width, the measured efficiency (again using the FW.02M criterion), and measured background spectra, the Minimum Detectable Activity (MDA) can be determined. We have used a $3 \sigma$ elevation above background within the FW.02M window centered on the peak, as the minimum detectable counting rate. This simple method yields the MDA table shown in Table 1 for a detector to source distance of $20 \mathrm{~cm}$. Table 2 presents MDA data for a detector to source distance of 1 meter. The various equations used to construct the entries are given at the end of the table. Note that the MDA values are specific to a distance and a collection time. In addition, the background used was taken at Santa Barbara, near sea level. Santa Barbara has mostly sedimentary rocks and clay soil. Lastly, these MDA values are specific to the 8-crystal array.

The MDA concept allows direct comparison between different detectors, at least for single sources and uncomplicated geometry. Table 3 presents some comparison data for common scintillators and the 8-crystal-CZT array. This comparison is slightly misleading in some cases, but provides a reference to start with. Figures 13 and 14 show one instance of a scintillator spectrum that would be difficult to interpret compared to similar data from the CZT array that is much more clear. In this case, where ${ }^{131} \mathrm{I}$ and ${ }^{239} \mathrm{Pu}$ are placed together, the usual 2 x $2 \mathrm{NaI}$ scintillator will produce a combined spectrum as shown in Figure 13. The ${ }^{131} \mathrm{I}+{ }^{239} \mathrm{Pu}$ peak shape is different from that of ${ }^{131} \mathrm{I}$ alone, but the difference is subtle. In Figure 14 the $414 \mathrm{keV}$ peak of ${ }^{239} \mathrm{Pu}$ is clearly seen and will permit identification of this isotope in the presence of ${ }^{131} \mathrm{I}$. Other ${ }^{131} \mathrm{I}$ peaks must be used to verify the presence of this isotope.

\subsection{2-crystal array}

We have combined the basic 8-crystal array with three identical units resulting in a 32-crystal array. In this detector we multiplexed each of the 16 pairs of CZT crystals into one MCA and recorded 16 individual spectra. These spectra were then gain and offset corrected, digitally summed, and the resulting spectrum used for isotope identification..

Representative spectra are presented in Figures 15 through 20, and an MDA table presented in Table 4. These data show some resolution degradation due partly to the summing process and partly due to poorly matched crystal pairs. Current technology would improve the overall results and will be demonstrated in August 2003.

\section{CONCLUSIONS}

It is possible to construct a portable gamma spectrometer using multiple CZT crystals. The 8-crystal detector has acceptable resolution and efficiency to be used for isotope identification in the field. An instrument constructed using this 8-crystal array has proven to be very rugged and readily capable of identifying the common medical, industrial, and SNM isotopes. MDA tables have been presented, based upon measured data using this instrument, that estimate MDA values for practical distances and collection times for hand-held instruments.

\section{ACKNOWLEDGEMENTS}

This work was supported by the U.S. Department of Energy, National Nuclear Security Administration Nevada Site Office, under Contract No. DE-AC08-96NV11718. 
Table 1. MDA for an 8-crystal CZT array at $20 \mathrm{~cm}$.

\begin{tabular}{|c|c|c|c|c|c|c|c|c|c|c|}
\hline \multirow[b]{2}{*}{ Isotope } & \multirow[b]{2}{*}{$\mathrm{keV}$} & \multicolumn{6}{|c|}{$\begin{array}{l}8 \text { Crystal CZT Data from } 12 \text { JAN 2003, Analysis from } 9 \text { energy points } \\
\text { Addspec used to sum all source spectra and all } 8 \text { background spectra }\end{array}$} & \multicolumn{3}{|c|}{$\begin{array}{l}\text { CZTMDA20cmAddspecBkg8Points } \\
\text { 28-May-03 }\end{array}$} \\
\hline & & yield & $\begin{array}{l}\text { Efficiency, } \\
\text { counts per gamma }\end{array}$ & $\begin{array}{l}3 \text { Sigma } \\
\text { Bkg, cps }\end{array}$ & $\begin{array}{l}\text { Air Absorption } \\
\text { for } 20 \mathrm{~cm}\end{array}$ & $\begin{array}{l}\text { at } 20 \mathrm{~cm}, 1 \mathrm{sec} \\
\text { MDA, Ci }\end{array}$ & $\begin{array}{c}\text { Specific } \\
\text { Activity, } \\
\text { Ci/g }\end{array}$ & $\begin{array}{l}\text { at } 20 \mathrm{~cm}, 1 \mathrm{sec} \\
\text { MDA,grams }\end{array}$ & $\begin{array}{l}\text { at } 20 \mathrm{~cm}, 100 \mathrm{sec} \\
\text { MDA, grams }\end{array}$ & $\begin{array}{l}\text { at } 20 \mathrm{~cm}, 100 \mathrm{sec} \\
\mathrm{MDA}, \mathrm{Ci}\end{array}$ \\
\hline \multirow[t]{4}{*}{ U-235 } & 143.786 & 0.105 & 0.1268 & 0.8578 & 0.9965 & 4.39E-06 & 2.16E-06 & $2.03 \mathrm{E}+00$ & 2.03E-01 & \\
\hline & 163.379 & 0.047 & 0.0889 & 0.7620 & 0.9967 & $1.24 \mathrm{E}-05$ & 2.16E-06 & $5.75 \mathrm{E}+00$ & $5.75 \mathrm{E}-01$ & \\
\hline & 185.739 & 0.53 & 0.0623 & 0.6635 & 0.9968 & $1.37 \mathrm{E}-06$ & $2.16 \mathrm{E}-06$ & 6.33E-01 & 6.33E-02 & \\
\hline & 205.333 & 0.047 & 0.0471 & 0.5876 & 0.9969 & $1.81 \mathrm{E}-05$ & 2.16E-06 & $8.36 \mathrm{E}+00$ & 8.36E-01 & \\
\hline \multirow[t]{4}{*}{ Pu-239 } & 203.52 & 0.0000056 & 0.0483 & 0.5942 & 0.9969 & $1.50 \mathrm{E}-01$ & 0.062 & $2.41 \mathrm{E}+00$ & $2.41 \mathrm{E}-01$ & \\
\hline & 344.94 & 0.0000057 & 0.0112 & 0.2984 & 0.9975 & $3.20 \mathrm{E}-01$ & 0.062 & $5.16 \mathrm{E}+00$ & $5.16 \mathrm{E}-01$ & \\
\hline & 375.018 & 0.0000158 & 0.0088 & 0.2791 & 0.9976 & $1.36 \mathrm{E}-01$ & 0.062 & $2.19 E+00$ & 2.19E-01 & \\
\hline & 413.691 & 0.0000151 & 0.0067 & 0.2666 & 0.9977 & $1.79 \mathrm{E}-01$ & 0.062 & $2.88 \mathrm{E}+00$ & $2.88 \mathrm{E}-01$ & \\
\hline \multirow[t]{3}{*}{ U-238 } & 295.22 & 0.189 & 0.0172 & 0.3574 & 0.9973 & 7.49E-06 & 3.35E-07 & $2.24 \mathrm{E}+01$ & $2.24 \mathrm{E}+00$ & \\
\hline & 351.99 & 0.363 & 0.0105 & 0.2929 & 0.9975 & $5.21 \mathrm{E}-06$ & 3.35E-07 & $1.56 \mathrm{E}+01$ & $1.56 \mathrm{E}+00$ & \\
\hline & 609.37 & 0.428 & 0.0023 & 0.2820 & 0.9980 & $1.95 \mathrm{E}-05$ & 3.35E-07 & $5.83 \mathrm{E}+01$ & $5.83 \mathrm{E}+00$ & \\
\hline \multirow[t]{2}{*}{ In-111 } & 171.28 & 0.9093 & 0.0780 & 0.7258 & 0.9967 & $6.98 \mathrm{E}-07$ & & & & $6.98 \mathrm{E}-08$ \\
\hline & 245.39 & 0.94 & 0.0287 & 0.4622 & 0.9971 & 1.17E-06 & & & & 1.17E-07 \\
\hline \multirow[t]{2}{*}{ Tl-201 } & 135.34 & 0.028 & 0.1500 & 0.9014 & 0.9964 & $1.46 \mathrm{E}-05$ & & & & $1.46 \mathrm{E}-06$ \\
\hline & 167.43 & 0.106 & 0.0831 & 0.7433 & 0.9967 & 5.75E-06 & & & & \\
\hline \multirow[t]{3}{*}{ Ga-67 } & 184.58 & 0.209 & 0.0634 & 0.6683 & 0.9968 & $3.44 \mathrm{E}-06$ & & & & 3.44E-07 \\
\hline & 208.95 & 0.0237 & 0.0449 & 0.5746 & 0.9970 & $3.68 \mathrm{E}-05$ & & & & $3.68 \mathrm{E}-06$ \\
\hline & 300.22 & 0.168 & 0.0164 & 0.3496 & 0.9974 & 8.64E-06 & & & & 8.64E-07 \\
\hline \multirow[t]{2}{*}{$\mathrm{I}-131$} & 284.3 & 0.0606 & 0.0191 & 0.3760 & 0.9973 & $2.21 \mathrm{E}-05$ & & & & $2.21 \mathrm{E}-06$ \\
\hline & 364.48 & 0.812 & 0.0096 & 0.2847 & 0.9975 & 2.49E-06 & & & & $2.49 \mathrm{E}-07$ \\
\hline Тc-99m & 140.511 & 0.8897 & 0.1352 & 0.8746 & 0.9965 & 4.96E-07 & & & & $4.96 \mathrm{E}-08$ \\
\hline Am-241 & 59.537 & 0.359 & 0.3218 & 1.1730 & 0.9951 & 6.93E-07 & & & & 6.93E-08 \\
\hline \multirow[t]{2}{*}{ Co-57 } & 122.06 & 0.856 & 0.1999 & 0.9724 & 0.9963 & $3.88 \mathrm{E}-07$ & & & & $3.88 \mathrm{E}-08$ \\
\hline & 136.47 & 0.1058 & 0.1466 & 0.8955 & 0.9964 & $3.94 \mathrm{E}-06$ & & & & $3.94 \mathrm{E}-07$ \\
\hline Cs-137 & 661.66 & 0.851 & 0.0018 & 0.2912 & 0.9980 & $1.28 \mathrm{E}-05$ & & & & $1.28 \mathrm{E}-06$ \\
\hline \multirow[t]{4}{*}{ Вa-133 } & 276.4 & 0.0717 & 0.0206 & 0.3909 & 0.9973 & $1.80 \mathrm{E}-05$ & & & & $1.80 \mathrm{E}-06$ \\
\hline & 302.85 & 0.1832 & 0.0160 & 0.3457 & 0.9974 & 8.03E-06 & & & & 8.03E-07 \\
\hline & 356.02 & 0.621 & 0.0102 & 0.2901 & 0.9975 & $3.11 \mathrm{E}-06$ & & & & $3.11 \mathrm{E}-07$ \\
\hline & 383.85 & 0.0894 & 0.0083 & 0.2752 & 0.9976 & 2.53E-05 & & & & 2.53E-06 \\
\hline
\end{tabular}

Efficiency in counts per gamma $=125090 * \mathrm{keV}^{\wedge}-2.7780$, excluding the 59 and $81 \mathrm{keV}$ points

3 sigma bkg $=3 *$ SQRT(bkg in counts/channel-second at a given keV*FW.02M in channels at the same keV), measured data used for $59 \mathrm{keV}$ point

bkg in counts/channel-sec $=\left(14.242+4330.89 /\left(1+\mathrm{e}^{\wedge}(-(\right.\right.$ channel-32.493) $\left./-216.23))\right) / 196860$ from 72 to $668 \mathrm{keV}$

FW.02M (in channels) $=0.1650 * \mathrm{keV}+20.68$

Energy calibration was: $\mathrm{keV}=0.2621 *$ channels- 2.8397

$\mathrm{MU}=0.905^{*} \mathrm{keV}^{\wedge}-0.382 \quad$ air attenuation $=\mathrm{EXP}\left(-\mathrm{MU}^{*} 20 * 0.001293\right)$ for $20 \mathrm{~cm}$

$\mathrm{MDA}$ at $20 \mathrm{~cm}$ in $\mathrm{Ci}=([3 \mathrm{sigma} \mathrm{bkg} / \mathrm{sqrt}$ (integration time) $] * 4 * \mathrm{pi}() * 20 \wedge 2 /($ Efficiency $*$ detector area* $3.7 \mathrm{E} 10 *$ yield $)) /[$ air attenuation $]$

detector area is $2 \mathrm{~cm}^{\wedge} 2$ for 8 crystals

MDA at $20 \mathrm{~cm}$ in grams $=$ MDA at $20 \mathrm{~cm}$ in $\mathrm{Ci} / \mathrm{Ci}$ per gram 
Table 2. MDA for an 8-crystal array at 1 meter.

\begin{tabular}{|c|c|c|c|c|c|c|c|c|c|c|}
\hline \multirow[b]{2}{*}{ Isotope } & \multirow[b]{2}{*}{$\mathrm{keV}$} & \multicolumn{7}{|c|}{$\begin{array}{l}8 \text { Crystal CZT Data from } 12 \text { JAN 2003, Analysis from } 9 \text { energy points } \\
\text { Addspec used to sum all source spectra and all } 8 \text { background spectra }\end{array}$} & \multicolumn{2}{|c|}{$\begin{array}{l}\text { CZTMDA1mAddspecBkg8Points } \\
\text { 28-May-03 }\end{array}$} \\
\hline & & yield & $\begin{array}{l}\text { Efficiency, } \\
\text { counts per gamma }\end{array}$ & $\begin{array}{l}3 \text { Sigma } \\
\text { Bkg, cps }\end{array}$ & $\begin{array}{l}\text { Air Absorption } \\
\quad \text { for } 1 \mathrm{~m}\end{array}$ & $\begin{array}{l}\text { at } 1 \mathrm{~m}, 1 \mathrm{sec} \\
\text { MDA, Ci }\end{array}$ & $\begin{array}{c}\text { Specific } \\
\text { Activity, } \\
\text { Ci/g }\end{array}$ & $\begin{array}{l}\text { at } 1 \mathrm{~m}, 1 \mathrm{sec} \\
\text { MDA,grams }\end{array}$ & $\begin{array}{l}\text { at } 1 \mathrm{~m}, 100 \mathrm{sec} \\
\text { MDA, grams }\end{array}$ & $\begin{array}{l}\text { at } 1 \mathrm{~m}, 100 \mathrm{sec} \\
\mathrm{MDA}, \mathrm{Ci}\end{array}$ \\
\hline \multirow[t]{4}{*}{ U-235 } & 143.786 & 0.105 & 0.1268 & 0.8578 & 0.9826 & $1.11 \mathrm{E}-04$ & $2.16 \mathrm{E}-06$ & $5.15 \mathrm{E}+01$ & $5.15 E+00$ & \\
\hline & 163.379 & 0.047 & 0.0889 & 0.7620 & 0.9834 & 3.15E-04 & 2.16E-06 & $1.46 \mathrm{E}+02$ & $1.46 \mathrm{E}+01$ & \\
\hline & 185.739 & 0.53 & 0.0623 & 0.6635 & 0.9842 & 3.47E-05 & $2.16 \mathrm{E}-06$ & $1.60 \mathrm{E}+01$ & $1.60 \mathrm{E}+00$ & \\
\hline & 205.333 & 0.047 & 0.0471 & 0.5876 & 0.9848 & 4.57E-04 & 2.16E-06 & $2.11 \mathrm{E}+02$ & $2.11 \mathrm{E}+01$ & \\
\hline \multirow[t]{4}{*}{ Pu-239 } & 203.52 & 0.0000056 & 0.0483 & 0.5942 & 0.9848 & $3.79 \mathrm{E}+00$ & 0.062 & $6.11 \mathrm{E}+01$ & $6.11 \mathrm{E}+00$ & \\
\hline & 344.94 & 0.0000057 & 0.0112 & 0.2984 & 0.9875 & $8.07 \mathrm{E}+00$ & 0.062 & $1.30 \mathrm{E}+02$ & $1.30 \mathrm{E}+01$ & \\
\hline & 375.018 & 0.0000158 & 0.0088 & 0.2791 & 0.9879 & $3.43 E+00$ & 0.062 & $5.54 \mathrm{E}+01$ & $5.54 \mathrm{E}+00$ & \\
\hline & 413.691 & 0.0000151 & 0.0067 & 0.2666 & 0.9884 & $4.51 \mathrm{E}+00$ & 0.062 & $7.27 \mathrm{E}+01$ & $7.27 \mathrm{E}+00$ & \\
\hline \multirow[t]{3}{*}{ U-238 } & 295.22 & 0.189 & 0.0172 & 0.3574 & 0.9868 & $1.89 \mathrm{E}-04$ & 3.35E-07 & $5.65 \mathrm{E}+02$ & $5.65 \mathrm{E}+01$ & \\
\hline & 351.99 & 0.363 & 0.0105 & 0.2929 & 0.9876 & 1.32E-04 & 3.35E-07 & $3.93 \mathrm{E}+02$ & $3.93 E+01$ & \\
\hline & 609.37 & 0.428 & 0.0023 & 0.2820 & 0.9899 & 4.92E-04 & 3.35E-07 & $1.47 \mathrm{E}+03$ & $1.47 \mathrm{E}+02$ & \\
\hline \multirow[t]{2}{*}{ In-111 } & 171.28 & 0.9093 & 0.0780 & 0.7258 & 0.9837 & $1.77 \mathrm{E}-05$ & & & & $1.77 \mathrm{E}-06$ \\
\hline & 245.39 & 0.94 & 0.0287 & 0.4622 & 0.9858 & 2.95E-05 & & & & 2.95E-06 \\
\hline \multirow[t]{2}{*}{ Tl-201 } & 135.34 & 0.028 & 0.1500 & 0.9014 & 0.9822 & $3.71 \mathrm{E}-04$ & & & & $3.71 \mathrm{E}-05$ \\
\hline & 167.43 & 0.106 & 0.0831 & 0.7433 & 0.9836 & $1.46 \mathrm{E}-04$ & & & & \\
\hline \multirow[t]{3}{*}{ Ga-67 } & 184.58 & 0.209 & 0.0634 & 0.6683 & 0.9842 & 8.71E-05 & & & & 8.71E-06 \\
\hline & 208.95 & 0.0237 & 0.0449 & 0.5746 & 0.9849 & $9.31 \mathrm{E}-04$ & & & & $9.31 \mathrm{E}-05$ \\
\hline & 300.22 & 0.168 & 0.0164 & 0.3496 & 0.9868 & $2.18 \mathrm{E}-04$ & & & & $2.18 \mathrm{E}-05$ \\
\hline \multirow[t]{2}{*}{ I-131 } & 284.3 & 0.0606 & 0.0191 & 0.3760 & 0.9866 & $5.60 \mathrm{E}-04$ & & & & $5.60 \mathrm{E}-05$ \\
\hline & 364.48 & 0.812 & 0.0096 & 0.2847 & 0.9878 & $6.30 \mathrm{E}-05$ & & & & $6.30 \mathrm{E}-06$ \\
\hline Tc-99m & 140.511 & 0.8897 & 0.1352 & 0.8746 & 0.9825 & $1.26 \mathrm{E}-05$ & & & & $1.26 \mathrm{E}-06$ \\
\hline Am-241 & 59.537 & 0.359 & 0.3218 & 1.1730 & 0.9757 & $1.77 \mathrm{E}-05$ & & & & $1.77 \mathrm{E}-06$ \\
\hline \multirow[t]{2}{*}{ Co-57 } & 122.06 & 0.856 & 0.1999 & 0.9724 & 0.9815 & $9.83 \mathrm{E}-06$ & & & & $9.83 \mathrm{E}-07$ \\
\hline & 136.47 & 0.1058 & 0.1466 & 0.8955 & 0.9823 & $\begin{array}{l}9.98 E-05 \\
\text { HעIV/U! }\end{array}$ & & & & $9.98 \mathrm{E}-06$ \\
\hline Cs-137 & 661.66 & 0.851 & 0.0018 & 0.2912 & 0.9903 & $3.21 \mathrm{E}-04$ & & & & $3.21 \mathrm{E}-05$ \\
\hline \multirow[t]{4}{*}{ Вa-133 } & 276.4 & 0.0717 & 0.0206 & 0.3909 & 0.9864 & $4.55 \mathrm{E}-04$ & & & & 4.55E-05 \\
\hline & 302.85 & 0.1832 & 0.0160 & 0.3457 & 0.9869 & 2.03E-04 & & & & 2.03E-05 \\
\hline & 356.02 & 0.621 & 0.0102 & 0.2901 & 0.9877 & 7.86E-05 & & & & 7.86E-06 \\
\hline & 383.85 & 0.0894 & 0.0083 & 0.2752 & 0.9880 & 6.38E-04 & & & & 6.38E-05 \\
\hline
\end{tabular}

Efficiency in counts per gamma $=125090 * \mathrm{keV}^{\wedge}-2.7780$, excluding the 59 and $81 \mathrm{keV}$ points

3 sigma bkg $=3 *$ SQRT(bkg in counts/channel-second at a given keV*FW.02M in channels at the same keV), measured data used for $59 \mathrm{keV}$ point

bkg in counts/channel-sec $=\left(14.242+4330.89 /\left(1+\mathrm{e}^{\wedge}(-(\right.\right.$ channel-32.493) $\left./-216.23))\right) / 196860$ from 72 to $668 \mathrm{keV}$

FW.02M (in channels) $=0.1650 * \mathrm{keV}+20.68$

Energy calibration was: $\mathrm{keV}=0.2621 *$ channels- 2.8397

$\mathrm{MU}=0.905 * \mathrm{keV} \wedge-0.382 \quad$ air attenuation $=\mathrm{EXP}(-\mathrm{MU} * 100 * 0.001293)$ for $1 \mathrm{~m}$

MDA at $1 \mathrm{~m}$ in $\mathrm{Ci}=([3$ sigma bkg / sqrt (integration time) $] * 4 * \mathrm{pi}() * 100 \wedge 2 /($ Efficiency $*$ detector area* $3.7 \mathrm{E} 10 *$ yield $)) /[$ air attenuation $]$

detector area is $2 \mathrm{~cm} \wedge 2$ for 8 crystals

MDA at $1 \mathrm{~m}$ in grams $=$ MDA at $1 \mathrm{~m}$ in $\mathrm{Ci} / \mathrm{Ci}$ per gram

Table 3. MDA comparison of several common detectors and the 8-crystal CZT array. The background was that of Santa Barbara and the source environment was clear of scattering objects. No combined sources are considered.

\begin{tabular}{|l|c|c|}
\hline \multirow{2}{*}{ Detector Type } & ${ }^{235}$ U Source, 100 Seconds Counting Time, 185 keV peak \\
\cline { 2 - 3 } & MDA @ 20 cm & MDA @ 3 meters \\
\hline \hline $2 \times 2 \mathrm{NaI}$ & $0.015 \mathrm{~g}$ & $3.6 \mathrm{~g}$ \\
\hline $3 \times 3 \mathrm{NaI}$ & $0.005 \mathrm{~g}$ & $1.1 \mathrm{~g}$ \\
\hline 8-crystal CZT array & $0.06 \mathrm{~g}$ & $14.9 \mathrm{~g}$ \\
\hline 20 detector JT-cooled HPGe array & & $0.66 \mathrm{~g}$ \\
\hline
\end{tabular}


Table 4. MDA for a 32-crystal array at 5 meters.

32 CRYSTAL CZT ARRAY at 5 METERS 12 OCT 2001

\begin{tabular}{|c|c|c|c|c|c|c|c|c|c|c|}
\hline Isotope & $\mathrm{keV}$ & yield & $\begin{array}{c}\text { Efficiency, } \\
\text { counts per gamma }\end{array}$ & $\begin{array}{c}5 \mathrm{~m} \\
3 \text { Sigma } \\
\text { Bkg, cps }\end{array}$ & $\begin{array}{l}\text { Air Absorption } \\
\text { for } 5 \mathrm{~m}\end{array}$ & $\begin{array}{c}\text { at } 5 \mathrm{~m}, 1 \mathrm{sec} \\
\mathrm{MDA}, \mathrm{Ci}\end{array}$ & $\begin{array}{c}\text { Specific } \\
\text { Activity, } \\
\text { Ci/g }\end{array}$ & $\begin{array}{l}\text { at } 5 \mathrm{~m}, 1 \mathrm{sec} \\
\text { MDA,grams }\end{array}$ & $\begin{array}{c}\text { at } 5 \mathrm{~m}, 100 \mathrm{sec} \\
\text { MDA, grams }\end{array}$ & $\begin{array}{c}\text { at } 5 \mathrm{~m}, 100 \mathrm{sec} \\
\mathrm{MDA}, \mathrm{Ci}\end{array}$ \\
\hline \multirow[t]{4}{*}{ U-235 } & 143.786 & 0.105 & 0.1582 & 2.1083 & 0.9160 & $1.47 \mathrm{E}-03$ & $2.16 \mathrm{E}-06$ & $6.80 \mathrm{E}+02$ & $6.80 \mathrm{E}+01$ & \\
\hline & 163.379 & 0.047 & 0.1126 & 1.9088 & 0.9199 & 4.16E-03 & $2.16 \mathrm{E}-06$ & $1.92 \mathrm{E}+03$ & $1.92 \mathrm{E}+02$ & \\
\hline & 185.739 & 0.53 & 0.0800 & 1.7274 & 0.9236 & 4.68E-04 & 2.16E-06 & $2.16 \mathrm{E}+02$ & $2.16 \mathrm{E}+01$ & \\
\hline & 205.333 & 0.047 & 0.0612 & 1.5977 & 0.9263 & 6.36E-03 & 2.16E-06 & $2.94 \mathrm{E}+03$ & $2.94 \mathrm{E}+02$ & \\
\hline \multirow[t]{4}{*}{ Pu-239 } & 203.52 & 0.0000056 & 0.0627 & 1.6088 & 0.9261 & $5.25 \mathrm{E}+01$ & 0.062 & $8.47 \mathrm{E}+02$ & $8.47 \mathrm{E}+01$ & \\
\hline & 344.94 & 0.0000057 & 0.0154 & 1.0671 & 0.9392 & $1.37 \mathrm{E}+02$ & 0.062 & $2.22 \mathrm{E}+03$ & $2.22 \mathrm{E}+02$ & \\
\hline & 375.018 & 0.0000158 & 0.0123 & 0.9998 & 0.9410 & $5.80 \mathrm{E}+01$ & 0.062 & $9.35 \mathrm{E}+02$ & $9.35 \mathrm{E}+01$ & \\
\hline & 413.691 & 0.0000151 & 0.0095 & 0.9263 & 0.9431 & $7.28 \mathrm{E}+01$ & 0.062 & $1.17 \mathrm{E}+03$ & $1.17 \mathrm{E}+02$ & \\
\hline \multirow[t]{3}{*}{ U-238 } & 295.22 & 0.189 & 0.0233 & 1.2045 & 0.9355 & $3.10 \mathrm{E}-03$ & 3.35E-07 & $9.27 \mathrm{E}+03$ & $9.27 \mathrm{E}+02$ & \\
\hline & 351.99 & 0.363 & 0.0146 & 1.0504 & 0.9396 & $2.24 \mathrm{E}-03$ & 3.35E-07 & $6.69 \mathrm{E}+03$ & $6.69 \mathrm{E}+02$ & \\
\hline & 609.37 & 0.428 & 0.0034 & 0.6853 & 0.9507 & $5.29 \mathrm{E}-03$ & 3.35E-07 & $1.58 \mathrm{E}+04$ & $1.58 \mathrm{E}+03$ & \\
\hline \multirow[t]{2}{*}{ In-111 } & 171.28 & 0.9093 & 0.0993 & 1.8399 & 0.9212 & 2.35E-04 & & & & 2.35E-05 \\
\hline & 245.39 & 0.94 & 0.0381 & 1.3908 & 0.9310 & 4.43E-04 & & & & 4.43E-05 \\
\hline \multirow[t]{3}{*}{ Tl-201 } & 80.3 & 0.208 & 0.7463 & 3.3175 & 0.8962 & 2.53E-04 & & & & 2.53E-05 \\
\hline & 135.34 & 0.028 & 0.1859 & 2.2100 & 0.9142 & 4.93E-03 & & & & 4.93E-04 \\
\hline & 167.43 & 0.106 & 0.1055 & 1.8727 & 0.9206 & 1.93E-03 & & & & \\
\hline \multirow[t]{4}{*}{ Ga-67 } & 93.31 & 0.379 & 0.5003 & 2.9516 & 0.9017 & 1.83E-04 & & & & 1.83E-05 \\
\hline & 184.58 & 0.209 & 0.0813 & 1.7359 & 0.9234 & $1.17 \mathrm{E}-03$ & & & & $1.17 \mathrm{E}-04$ \\
\hline & 208.95 & 0.0237 & 0.0585 & 1.5762 & 0.9268 & $1.30 \mathrm{E}-02$ & & & & $1.30 \mathrm{E}-03$ \\
\hline & 300.22 & 0.168 & 0.0223 & 1.1888 & 0.9359 & $3.60 \mathrm{E}-03$ & & & & 3.60E-04 \\
\hline \multirow[t]{2}{*}{ Xe-133 } & 30.75 & 0.379 & 0.4018 & 7.0020 & 0.8538 & 5.72E-04 & & & & 5.72E-05 \\
\hline & 81 & 0.371 & 0.7292 & 3.2952 & 0.8966 & $1.44 \mathrm{E}-04$ & & & & $1.44 \mathrm{E}-05$ \\
\hline \multirow[t]{3}{*}{$\mathrm{I}-131$} & 80.18 & 0.0262 & 0.7492 & 3.3214 & 0.8962 & $2.00 \mathrm{E}-03$ & & & & $2.00 \mathrm{E}-04$ \\
\hline & 284.3 & 0.0606 & 0.0257 & 1.2403 & 0.9346 & 9.03E-03 & & & & 9.03E-04 \\
\hline & 364.48 & 0.812 & 0.0133 & 1.0223 & 0.9404 & $1.07 \mathrm{E}-03$ & & & & $1.07 \mathrm{E}-04$ \\
\hline \multirow[t]{2}{*}{$\mathrm{I}-125$} & 27.5 & 1.395 & 0.3005 & 7.6379 & 0.8479 & $2.28 \mathrm{E}-04$ & & & & $2.28 \mathrm{E}-05$ \\
\hline & 35.49 & 0.0667 & 0.3452 & 6.2628 & 0.8610 & 3.35E-03 & & & & 3.35E-04 \\
\hline Tc-99m & 140.511 & 0.8897 & 0.1682 & 2.1464 & 0.9153 & $1.66 \mathrm{E}-04$ & & & & $1.66 \mathrm{E}-05$ \\
\hline Am-241 & 59.537 & 0.359 & 1.0130 & 4.1872 & 0.8844 & $1.38 \mathrm{E}-04$ & & & & $1.38 \mathrm{E}-05$ \\
\hline \multirow[t]{2}{*}{ Co-57 } & 122.06 & 0.856 & 0.2447 & 2.3949 & 0.9109 & 1.33E-04 & & & & 1.33E-05 \\
\hline & 136.47 & 0.1058 & 0.1818 & 2.1957 & 0.9144 & 1.33E-03 & & & & 1.33E-04 \\
\hline Cs-137 & 661.66 & 0.851 & 0.0027 & 0.6428 & 0.9522 & $3.10 \mathrm{E}-03$ & & & & $3.10 \mathrm{E}-04$ \\
\hline \multirow[t]{5}{*}{ Ba-133 } & 80.997 & 0.232 & 0.7293 & 3.2953 & 0.8966 & 2.31E-04 & & & & $2.31 \mathrm{E}-05$ \\
\hline & 276.4 & 0.0717 & 0.0278 & 1.2678 & 0.9340 & 7.24E-03 & & & & 7.24E-04 \\
\hline & 302.85 & 0.1832 & 0.0218 & 1.1808 & 0.9362 & 3.36E-03 & & & & $3.36 \mathrm{E}-04$ \\
\hline & 356.02 & 0.621 & 0.0141 & 1.0411 & 0.9399 & $1.34 \mathrm{E}-03$ & & & & $1.34 \mathrm{E}-04$ \\
\hline & 383.85 & 0.0894 & 0.0116 & 0.9819 & 0.9415 & $1.07 \mathrm{E}-02$ & & & & $1.07 \mathrm{E}-03$ \\
\hline
\end{tabular}

Efficiency $=88136 * \mathrm{keV}^{\wedge}-2.663 \mathrm{c} /$ gamma $>80 \mathrm{keV}$

3 sigma bkg $=52.033 * \mathrm{SQRT}(\#$ of detectors $/ 8) * \mathrm{keV}^{\wedge}-0.7782$ for the IDD array at zero feet

bkg at $5 \mathrm{~m}=($ bkg at zero feet $* \operatorname{EXP}(-0.0020 * 5 * 39.37 / 12)$

$\mathrm{MU}=0.905 * \mathrm{keV}^{\wedge}-0.382 \quad$ air attenuation $=\mathrm{EXP}\left(-\mathrm{MU}^{*} 500 * 0.001293\right)$ for $5 \mathrm{~m}$

MDA at $5 \mathrm{~m}$ in $\mathrm{Ci}=([3$ sigma bkg / sqrt (integration time) $] * 4 * \mathrm{pi}() * 500 \wedge 2 /($ Efficiency $*$ detector area* 3.7 E10 * yield) $) /[$ air attenuation $]$

detector area is $8 \mathrm{~cm} \wedge 2$ for 32 crystals

MDA at $20 \mathrm{~cm}$ in grams $=$ MDA at $20 \mathrm{~cm}$ in $\mathrm{Ci} / \mathrm{Ci}$ per gram 


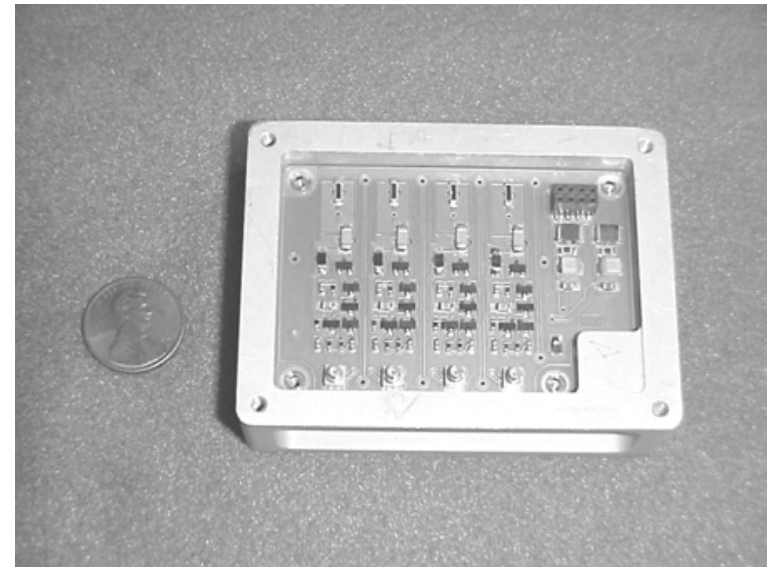

Figure 1. Assembled 8-crystal detector module.

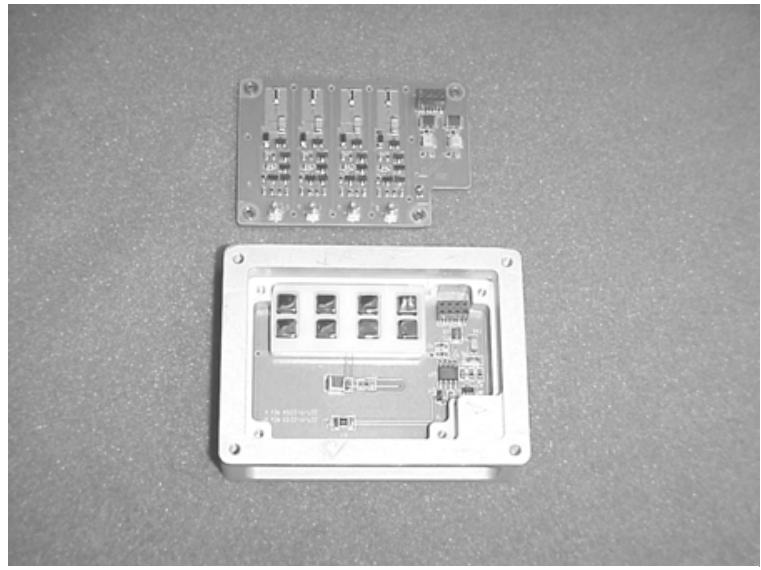

Figure 2. Interior view of 8-crystal detector module. Analog board removed, 8 CZT crystals in place on high-voltage board.

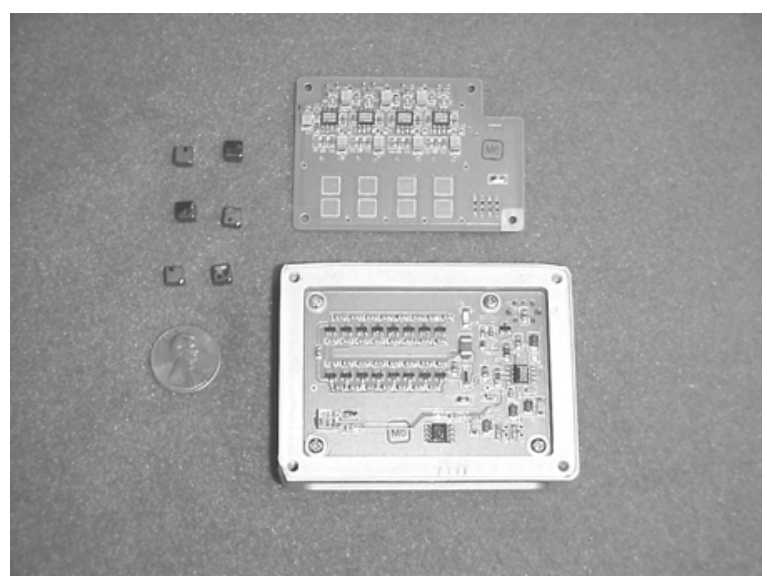

Figure 3. High-voltage board side of detector module. Six CZT crystals at one side, and bottom of analog board at top.

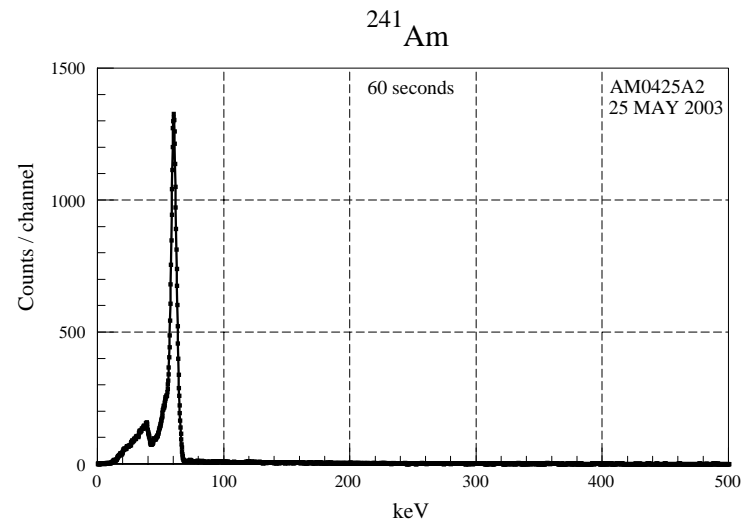

Figure 4. ${ }^{241}$ Am spectrum, 8-crystal array.

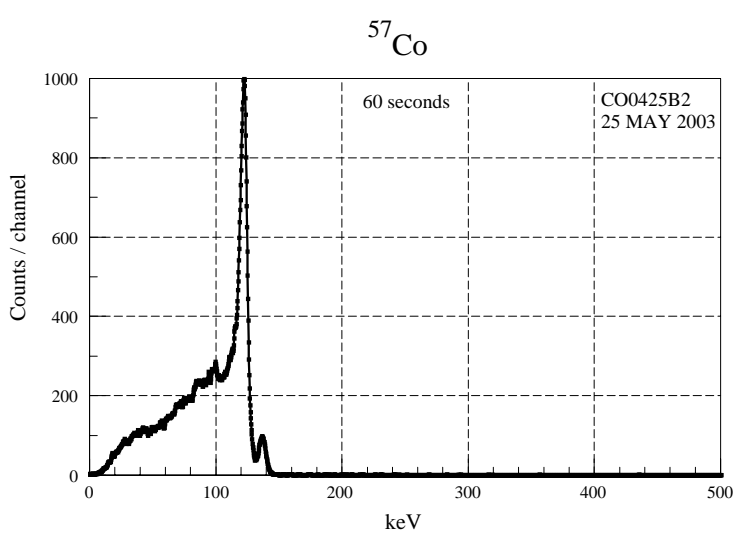

Figure 5. ${ }^{57}$ Co spectrum, 8-crystal array. 


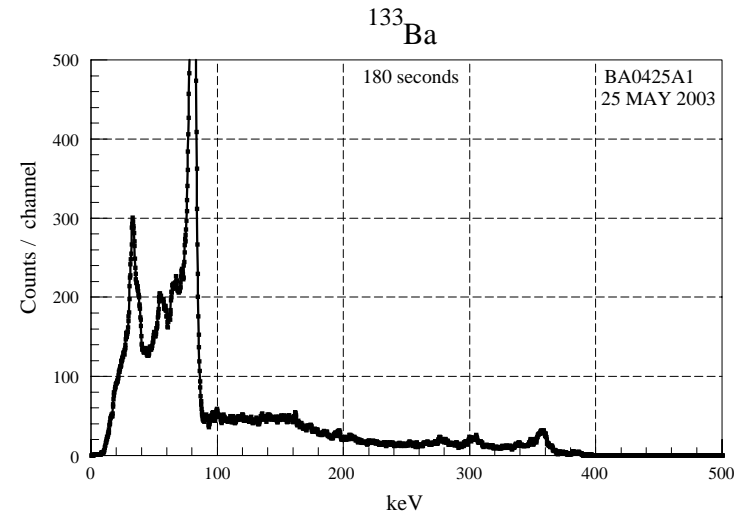

Figure 6. ${ }^{133} \mathrm{Ba}$ spectrum, 8-crystal array.

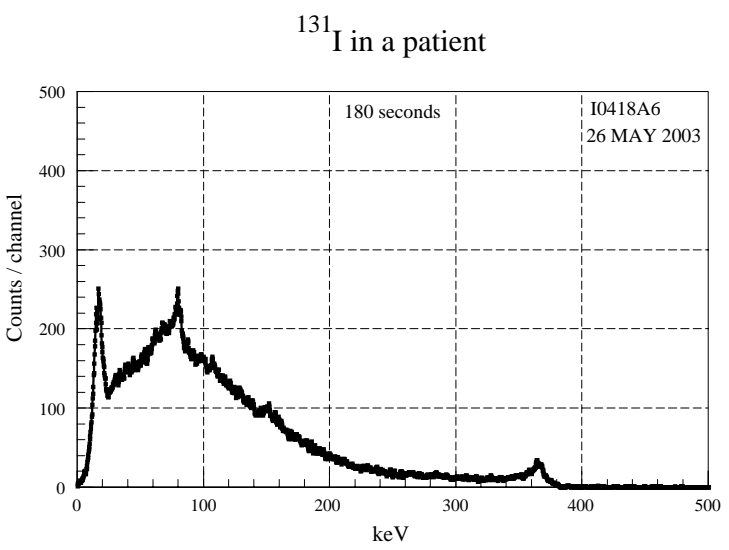

Figure 8. ${ }^{131}$ I spectrum in a patient, 8-crystal array.

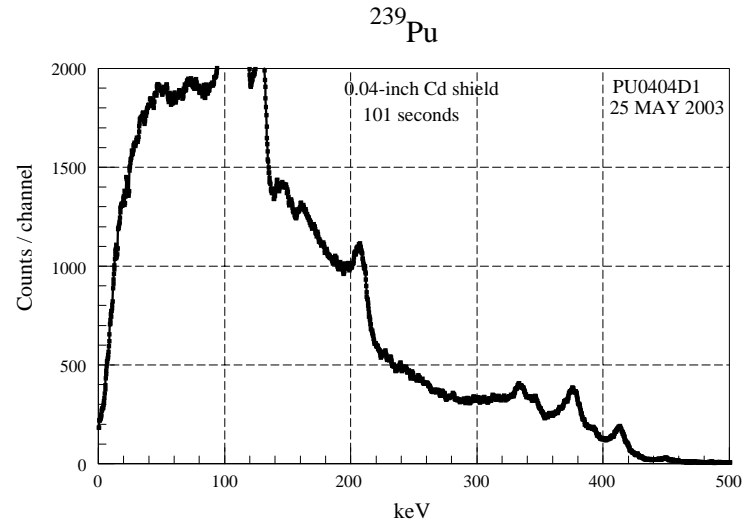

Figure 10. ${ }^{239} \mathrm{Pu}$ spectrum from 6 grams of metallic plutonium, 8-crystal array.

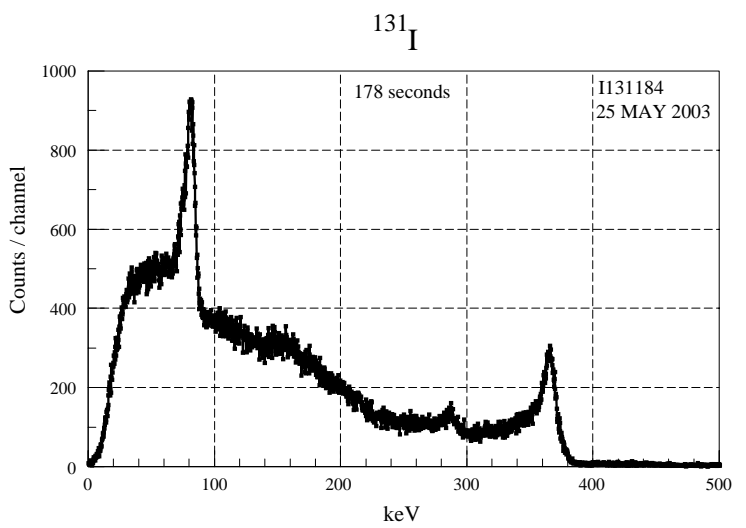

Figure 7. ${ }^{131}$ I spectrum, 8-crystal array.

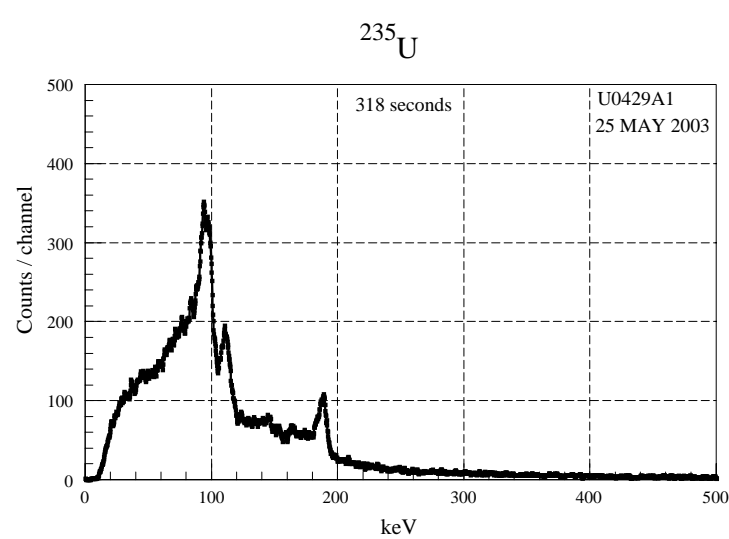

Figure 9. ${ }^{235} \mathrm{U}$ spectrum from LEU fuel pellets, 8-crystal array.

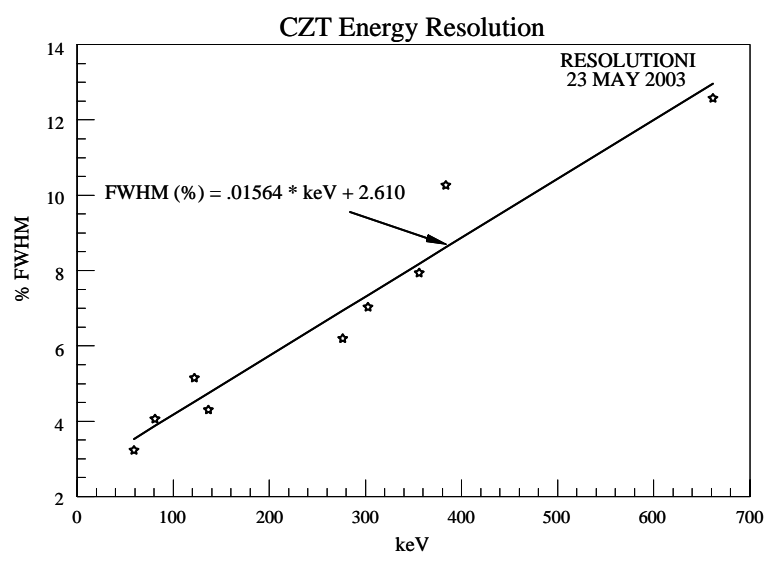

Figure 11. Energy resolution of 8-crystal array. 


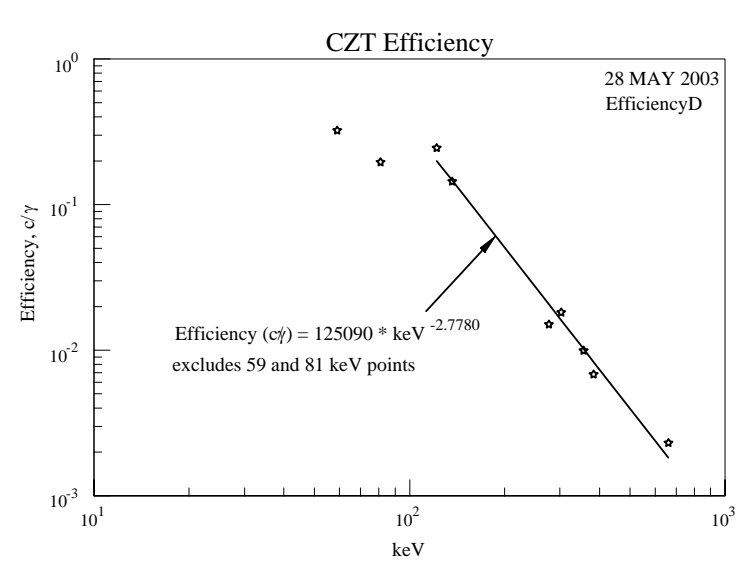

Figure 12. Efficiency of 8-crystal array.

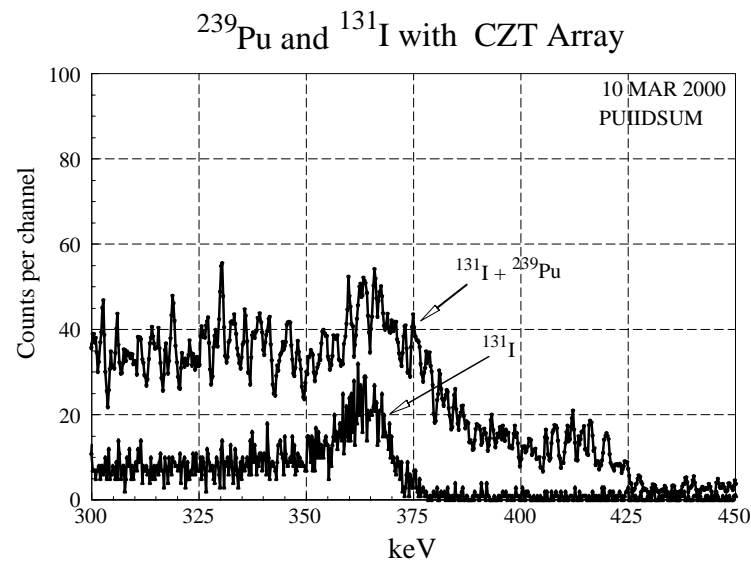

Figure 14. Comparison of 8-crystal CZT array spectra for ${ }^{131} \mathrm{I}$ and ${ }^{131} \mathrm{I}$ plus ${ }^{239} \mathrm{Pu}$ sources. The $414 \mathrm{keV}$ peak from ${ }^{239} \mathrm{Pu}$ clearly separates this component of the combination.

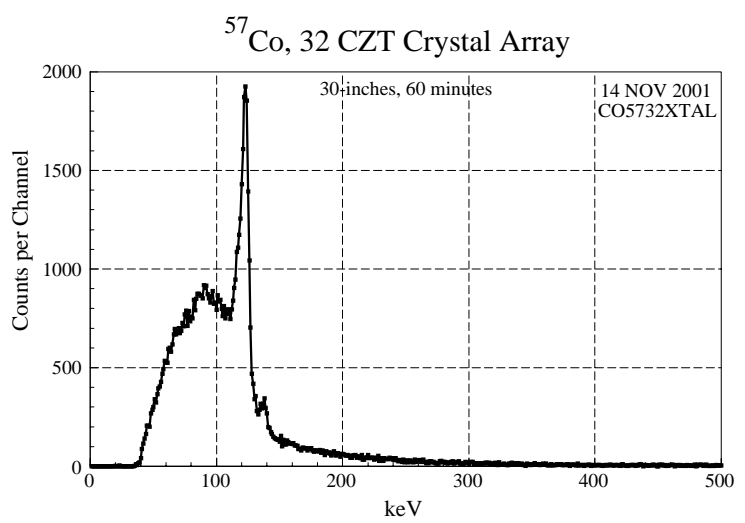

Figure 16. ${ }^{57}$ Co spectrum, 32-crystal array.

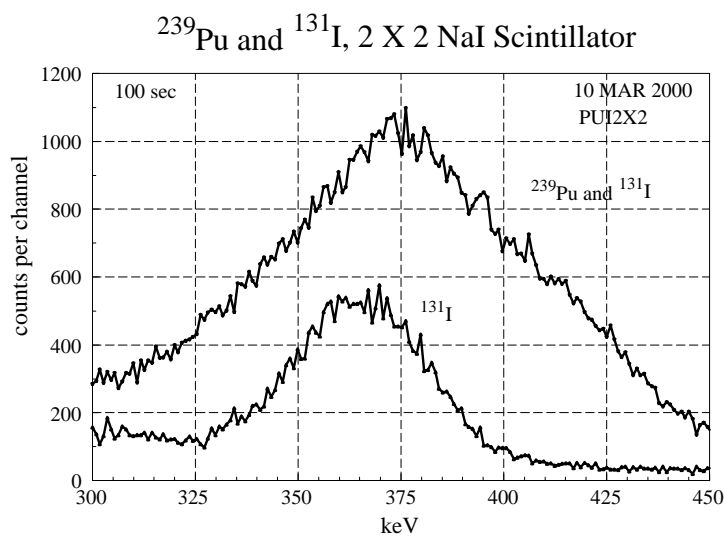

Figure 13. Comparison of $2 \times 2$ NaI spectra for ${ }^{131} \mathrm{I}$ and ${ }^{131} \mathrm{I}$ plus ${ }^{239} \mathrm{Pu}$ sources. It is not readily apparent that the combination of sources could be identified as separate components.

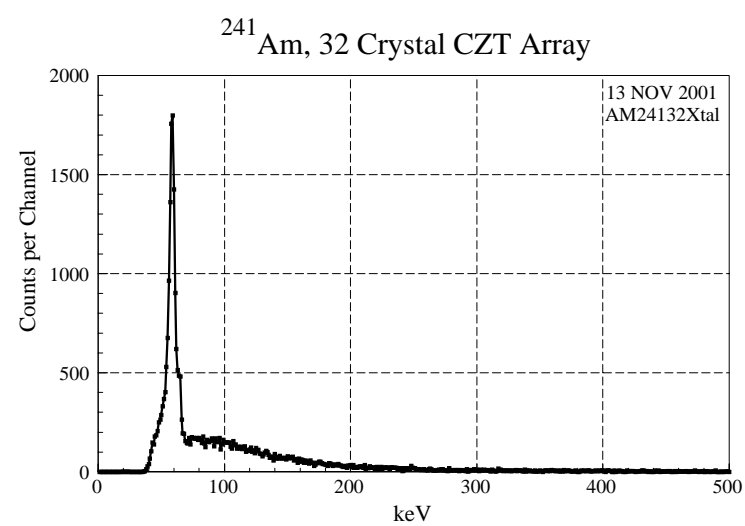

Figure 15. ${ }^{241} \mathrm{Am}$ spectrum, 32-crystal array.

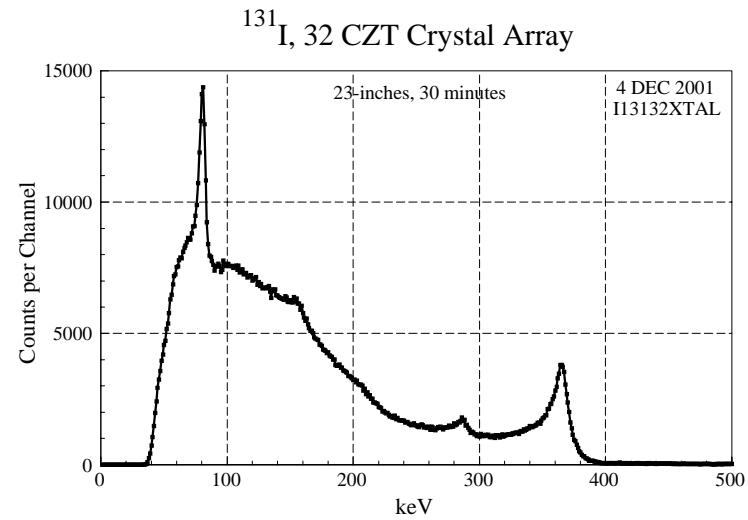

Figure 17. ${ }^{131}$ I spectrum, 32-crystal array. 


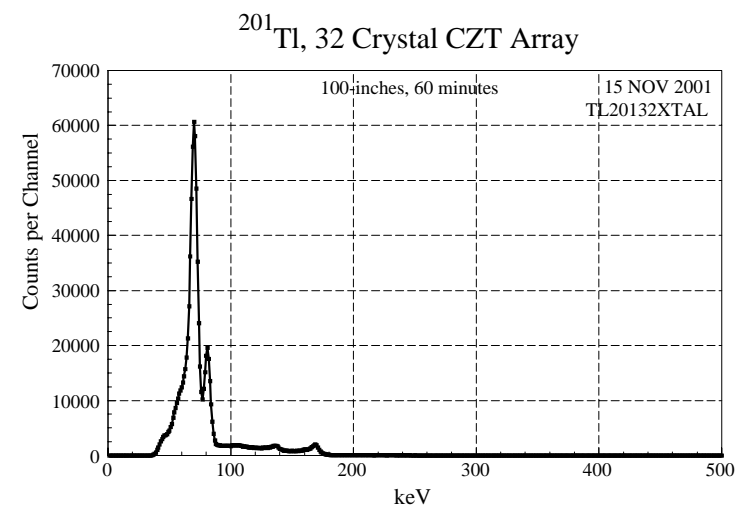

Figure 18. ${ }^{201} \mathrm{Tl}$ spectrum, 32-crystal array.

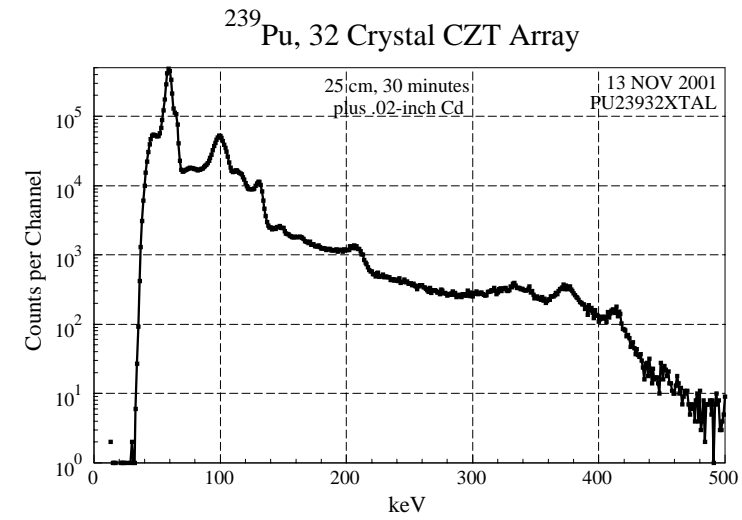

Figure $20 \cdot{ }^{239} \mathrm{Pu}$ spectrum, 32-crystal array, log scale.

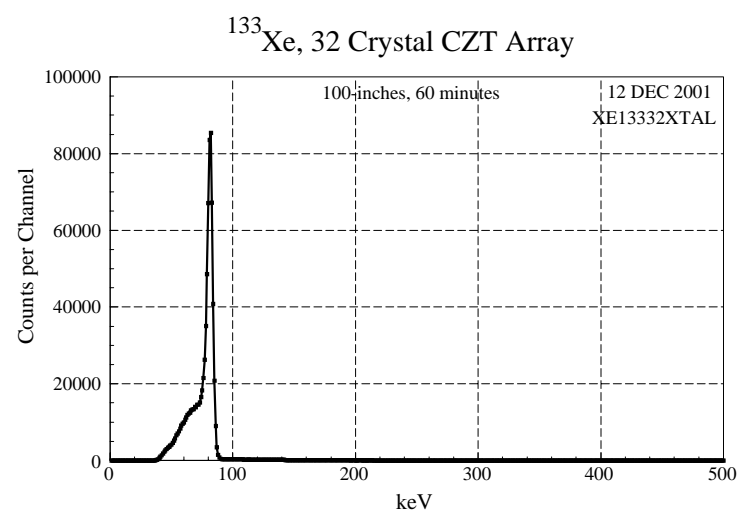

Figure 19. ${ }^{133}$ Xe spectrum, 32-crystal array. 


\section{DISTRIBUTION LIST}

U.S. Department of Energy

National Nuclear Security Administration

Nevada Site Office

Technical Library

P.O. Box 98518

Las Vegas, NV 89193-8518

U.S. Department of Energy

National Nuclear Security Administration

Nevada Site Office

Nuclear Testing Archive

P.O. Box 98518

Las Vegas, NV 89193-8518

U.S. Department of Energy

Office of Scientific and Technical Information

P.O. Box 62

Oak Ridge, TN 37831-0062 